\title{
Mortalidade domiciliar de idosos no município do Rio de Janeiro durante a pandemia de Coronavírus, 2020
}

\author{
Home deaths of older people in the city of Rio de Janeiro during the Coronavirus pandemic, 2020
}

\author{
Dalia Elena Romero' $\mathbb{I D}$ \\ Jessica Muzy' (ID) \\ Débora Castanheiral (ID \\ Aline Pinto Marques' ${ }^{1}$ \\ Nathália Andrade de Souza ${ }^{2}$ (D)
}

\section{Resumo}

Objetivo: analisar os óbitos ocorridos no município do Rio de Janeiro (RJ, Brasil) segundo local de ocorrência, faixa etária, causa e características sociodemográficas no contexto da pandemia de Covid-19. Método: calculou-se a distribuição da mortalidade por local de ocorrência, faixa etária e causa. O "excesso de mortalidade" foi analisado pela comparação das médias mensais dos óbitos por local de ocorrência, causas e características sociodemográficas nos meses de abril a junho dos anos 2017, 2018 e 2019, com aqueles ocorridos nos mesmos meses de 2020. Resultados: os óbitos domiciliares aumentaram quando comparada com a média no triênio anterior. As principais causas de mortalidade não foram alteradas, mas tiveram aumentos relevantes. Destaca-se o crescimento da insuficiência respiratória não classificada e dos óbitos por causa mal definida. Quanto às características sociodemográficas, verificou-se aumento maior entre homens, raça/ cor negra, viúvos e baixa escolaridade. Conclusão: o aumento da mortalidade domiciliar encontrado no município do Rio de Janeiro pode estar associado a efeitos da pandemia de Covid-19. Além disso, o aumento de mortes com causa básica mal definida pode estar associado à pandemia de Covid-19 em razão da falta de testes e dificuldade de acesso a serviços de saúde. A maior vulnerabilidade dos idosos é conhecida, porém estudos adicionais são importantes para entender os diferenciais de sexo e estado civil. A ligação entre raça/cor negra e menor nível de escolaridade e maior chance de mortalidade domiciliar ocorre em razão de uma sobreposição de riscos durante a vida, que leva pessoas nesses grupos à maior vulnerabilidade.

\footnotetext{
Fundação Oswaldo Cruz, Instituto de Comunicação e Informação Científica e Tecnológica em Saúde (ICICT/Fiocruz). Rio de Janeiro, RJ, Brasil.

2 Universidade Federal do Rio de Janeiro, Instituto de Filosofia e Ciências Sociais (IFCS/UFRJ). Rio de Janeiro, RJ, Brasil.
}

Os autores declaram não haver conflito na concepção deste trabalho.

Não houve financiamento para a execução deste trabalho.

Correspondência/Correspondence

Dalia Elena Romero

Palavras-chave: Pandemias. Infecções por Coronavirus. Mortalidade. Saúde do Idoso. 


\section{Abstract}

Objective: to analyze deaths in the city of Rio de Janeiro (RJ, Brazil) according to the place of occurrence, age group, cause, and sociodemographic characteristics in the context of the Covid-19 pandemic. Method: the distribution of mortality by place of occurrence, age group, and the cause was calculated. The "excess mortality" was analyzed by comparing the monthly averages of deaths by place of occurrence, causes, and sociodemographic characteristics in April to June of the years 2017, 2018, and 2019 with those that occurred in the same months of 2020. Results: home deaths increased when compared to the average in the previous triennium. The main causes of mortality were not altered but had significant increases. The growth of unclassified respiratory failure and deaths due to ill-defined causes is emphasized. Regarding the sociodemographic characteristics, there was a greater increase among men of black race/color, widowers, and with low education. Conclusion: the increased home deaths found in the city of Rio de Janeiro may be associated with the effects of the Covid-19 pandemic. Besides, the increase in deaths due to ill-defined causes may be associated with the Covid-19 pandemic due to the lack of tests and difficulty in accessing health services. The greater vulnerability of older people is known, but additional studies are important to understand the gender and marital status differences. Black race/color and a lower level of education are associated with a higher chance of home mortality due to an overlap of risks throughout life, leading people in these groups to greater vulnerability.

\section{INTRODUÇÃO}

Desde o dia 6 de março de 2020, quando se registrou o primeiro caso de Covid-19 no Estado do Rio de Janeiro, o risco de morte pela doença aumentou aceleradamente. Em outubro de 2020 o Estado tinha a segunda maior taxa de mortalidade por Covid-19 (117,5 por 100.000 habitantes, segundo dados do Monitora COVID-19, da Fundação Osvaldo Cruz) ${ }^{1}$, tendo acumulado até 27 de outubro, 20.292 óbitos por essa causa, sendo 14.797 de pessoas idosas (72,9\%). No município do Rio de Janeiro ocorreram 11.952 óbitos, sendo 9.179 (76,8\%) de pessoas com 60 anos ou mais ${ }^{2}$.

A taxa de letalidade de pessoas idosas é especialmente relevante no estado do Rio de Janeiro, uma vez que seu percentual de idosos na população é o segundo maior do país (16,3\%), ficando atrás apenas do Rio Grande do Sul (17,6\%). No município a proporção de idosos é de $14 \%{ }^{2}$.

Juntamente com o aumento de mortes provocado pela Covid-19, também é possível observar uma ampliação da mortalidade domiciliar, problema que ganha destaque com a situação de pandemia observada no país ${ }^{3,4}$. Apesar do fenômeno ter sido apontado, suas causas e seus significados para a mortalidade de pessoas idosas ainda são pouco analisados.

É importante destacar que o óbito domiciliar não aponta necessariamente para falhas na atenção à saúde. Quando ocorre em por causas crônicodegenerativas, sendo acompanhado de cuidados paliativos $^{5}$, com uma boa rede de suporte, com orientação especializada, num ambiente tranquilo e confortável, sem dor física e em contato com entes queridos, o óbito no domicílio pode ser indicador de uma morte digna e humanizada, uma "boa morte". Contudo, não é isso que ocorre no caso de morte por doença altamente transmissível, que frequentemente demanda internação para tratamento, como é o caso da Covid-19, os óbitos que ocorrem no domicílio evidenciam desassistência, lacunas no sistema de saúde e na assistência social, assim como carência de atenção oportuna e preventiva que deveria ser ofertada pela Atenção Primária à Saúde (APS) ${ }^{4}$.

Outro aspecto importante quando analisamos a mortalidade domiciliar durante a pandemia de Covid-19 é o impacto que a alta das internações por essa causa pode ter sobre o panorama da mortalidade domiciliar, tanto em relação ao conjunto de causas de mortalidade quanto na sua quantidade. 
Todos esses aspectos são ainda mais relevantes quando tratamos de residências coletivas de pessoas idosas, como as Instituições de Longa Permanência para Idosos (ILPI), onde o adoecimento por Covid-19 pode representar um grande risco de infecção para outras pessoas institucionalizadas, e trabalhadores ${ }^{7,8}$ e onde estão concentrados vários idosos em estado frágil e vulnerável, que têm maior possibilidade de ser afetados pela diminuição dos leitos disponíveis para internação em razão de sua reversão para o tratamento do coronavírus.

Ademais, as desigualdades de acesso ao sistema de saúde e as diferenças dentro do território municipal trazem elemento ainda mais desafiador ao cenário de combate à pandemia no Rio de Janeiro, ${ }^{90}$. Portanto, a análise das causas de mortalidade domiciliar por local de ocorrência e por marcadores sociodemográficos é essencial para a compreensão da evolução da pandemia no Estado e município.

Diante do considerado, este artigo tem como objetivo analisar os óbitos ocorridos no município do Rio de Janeiro segundo local de ocorrência, faixa etária, causa e características sociodemográficas no contexto da pandemia de Covid-19.

\section{MÉTODO}

O universo do estudo foram todos os óbitos ocorridos no município do Rio de Janeiro entre 2010 e 2020, segundo local de ocorrência, faixa etária e causa do óbito. Utilizou-se os dados da Declaração de Óbito (DO) consolidados no Sistema de Informação em Mortalidade (SIM) do Rio de Janeiro e disponibilizados online pela Secretaria Municipal de Saúde e Secretaria de Saúde do Governo do Estado do Rio de Janeiro ${ }^{11}$. Embora seja possível obter os dados mais recentes, a escolha por analisar somente o período de abril a junho se justifica pela qualidade dos dados. Espera-se que a não inclusão dos períodos mais recentes evite potenciais falhas ou atrasos de registro de óbitos. Esses problemas podem ocorrer dada a necessidade de revisão dos casos pelas secretarias estaduais e municipais de saúde. Os dados foram atualizados até 15 de outubro de 2020.

A classificação do local de ocorrência foi feita de acordo com a DO, tendo as seguintes opções:
1. Hospital; 2. Outros estabelecimentos de saúde; 3. Domicílio; 4. Via pública; 5. Outro (se não os já listados) e 6. Ignorado (opção utilizada quando não é possível identificar o local de ocorrência do óbito). Ressalta-se que a DO não permite identificar o tipo de domićlilio (particular ou coletivo), inviabilizando a identificação de óbitos em ILPI, por exemplo.

A identificação de alterações no local de ocorrência e grupo etário dos óbitos foi feita através da análise do número e proporção de óbitos gerais e em domicílios, segundo faixa etária e ano no município do Rio de Janeiro, entre 2010 e 2020. Foram consideradas 4 faixas etárias para a análise: menos de 30 anos, de 30 a 59 anos, 60 a 79 anos e 80 anos ou mais.

Utilizou-se a medida de "excesso de mortalidade" de idosos como uma forma de identificar os óbitos atribuíveis à Covid-19. Para estimar o "excesso de mortalidade" de idosos no período da pandemia, no município do Rio de Janeiro, foram comparados os óbitos de pessoas de 60 anos ou mais entre abril a junho de 2020 com a média mensal de mortes do mesmo grupo etário, nos mesmos meses de ocorrência, dos três anos anteriores (2017, 2018 e 2019) segundo local do óbito. Em seguida, estimouse a diferença percentual entre a mortalidade em 2020 e a média mensal dos três anos anteriores. Essa diferença indica o "excesso de mortalidade" em 2020.

A estimativa do excesso da mortalidade para a população idosa no período também foi realizada segundo causas, de acordo com a $10^{a}$ Revisão da Classificação Internacional de Doenças (CID-10) para óbitos ocorridos em domić́lio. Essa análise foi aprofundada pela estimativa da proporção de óbitos de idosos com causa básica mal definida (capítulo XVIII da CID-10) que ocorreram em domicílio, mês a mês, comparando os mesmos meses do triênio de 20172019 e 2020, no município do Rio de Janeiro. Esse indicador é uma medida utilizada com frequência para a avaliação da qualidade do registro da causa básica de óbito e da qualidade da atenção, visto que reflete também a estrutura para diagnóstico disponível.

Por fim, a distribuição sociodemográfica dos óbitos de idosos segundo local de ocorrência (domić́lio ou óbitos gerais) foi analisada no mesmo período a partir das variáveis disponíveis no sistema, sendo elas: sexo, raça/cor, estado civil e escolaridade. 
Todos os dados utilizados são dados secundários de acesso público disponíveis no SIM. Dispensando a necessidade de apreciação ao Comitê de Ética em Pesquisa, segundo a legislação vigente.

\section{RESULTADOS}

Na Figura 1 é possível observar a diferença na distribuição por idade do número de óbitos por local de ocorrência. Para óbitos domiciliares a faixa etária predominante é a de 80 anos e mais, enquanto que para óbitos em todos os locais de ocorrência a faixa entre 60 e 79 anos de idade apresenta número mais alto. Verifica-se também que a proporção de óbitos que ocorrem em domicílio aumenta de forma expressiva para o grupo de 80 anos e mais.

A Tabela 1 trata dos óbitos de pessoas idosas no Estado e Município do Rio de Janeiro segundo local de ocorrência. É possível identificar que o Estado do Rio de Janeiro teve um excesso de mortalidade geral de 9.215 idosos, o que representa, um incremento de 36,5\% em relação ao mesmo período do ano anterior. Vale ressaltar que $57 \%$ dos óbitos do estado se concentram no município do Rio de Janeiro $(n=7,023)$.

Ao comparar a diferença entre o estado e o município do Rio de Janeiro, observa-se que em 2020 o município teve um aumento de $78,2 \%$ dos óbitos domiciliares, em relação à média do triênio anterior. No estado esse crescimento foi de $54,3 \%$. Em relação aos óbitos ocorridos em outro estabelecimento de saúde, a diferença no período foi de 78,1\% no município e $46,5 \%$ no estado.

A Tabela 2 mostra a mortalidade domiciliar de pessoas de 60 anos ou mais ocorridas no município do Rio de Janeiro segundo a causa do óbito. A mortalidade em 2020 apresenta aumento para todos os grupos de causas (diferença percentual de $78 \%$ ). A causa de mortalidade com maior diferença absoluta de óbitos no período foi a hipertensão, passando de 153 para 364 óbitos em 2020, um excesso de mortalidade de $137 \%$. Outra causa com aumento significativo no número de óbitos domiciliares foi a diabetes, com $91,7 \%$ a mais que o período anterior.

As principais causas de morte nos domicílios nos meses de junho a abril do triênio 2017-2019 foram as doenças do aparelho circulatório, neoplasias, diabetes e doenças do aparelho respiratório. Esse cenário não se modifica em 2020. Apesar disso, é possível observar grande crescimento das mortes por doenças infecciosas e parasitarias (725\%), além do registro de 76 óbitos relacionados a doenças por vírus de localização não especificada (que nos meses analisados entre 2017 e 2019 não apresentaram casos). Entre as doenças do aparelho circulatório, que cresceram $12 \%$, destaca-se o crescimento de $400 \%$ da insuficiência respiratória não classificada em outra parte.

Os óbitos cuja causa foi registrada como do capítulo XVIII (sintomas, sinais e achados anormais de exames clínicos e de laboratório, não classificados em outra parte) passaram de $8,7 \%$ nos meses analisados do triênio anterior para 20,1\% em 2020. Em números absolutos, na média dos meses considerados do triênio anterior havia sido registrado um total de 152 óbitos, que passa para 662 em 2020, um aumento de $335,5 \%$.

Na Tabela 3 pode-se observar número, proporção e diferença percentual de óbitos gerais e ocorridos em domicílios nos meses de abril a junho do triênio 2017-2019 e 2020, segundo características sociodemográficas. Tanto quanto aos óbitos em domicílio quanto em outros locais foi observado maior aumento percentual entre os homens em relação às mulheres. Quanto à raça/cor, observouse que a população idosa negra teve maior excesso de mortalidade nos domicílios que a população branca no período, $109 \%$ e $73,9 \%$, respectivamente. O excesso de óbitos domiciliares de idosos pardos em 2020 foi similar ao de idosos identificados como brancos, sendo levemente superior $(77,7 \%)$.

Destaca-se ainda o aumento expressivo dos óbitos sem raça/cor informada, especialmente na mortalidade domiciliar. 


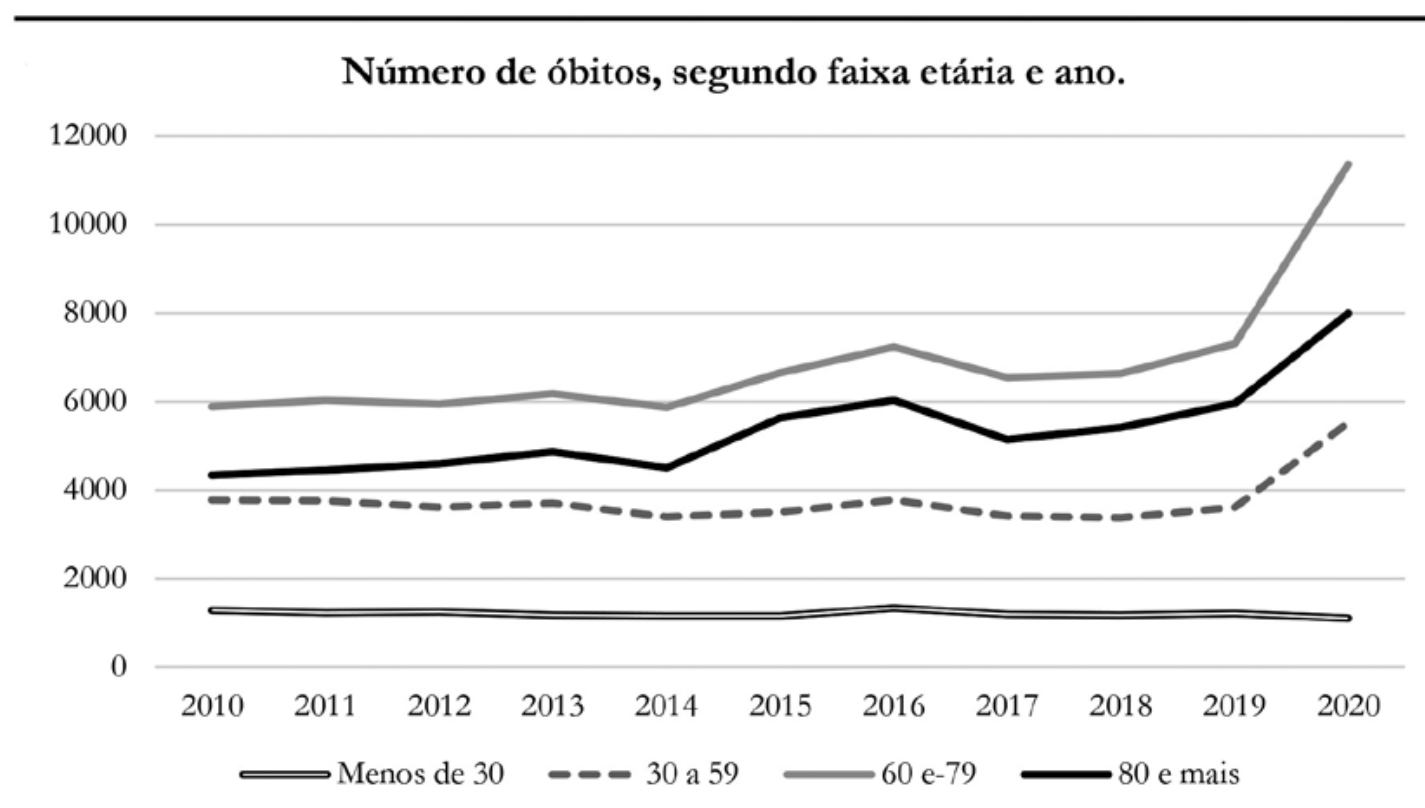

Número de óbitos que ocorreram em domicílio, segundo faixa etária e ano.

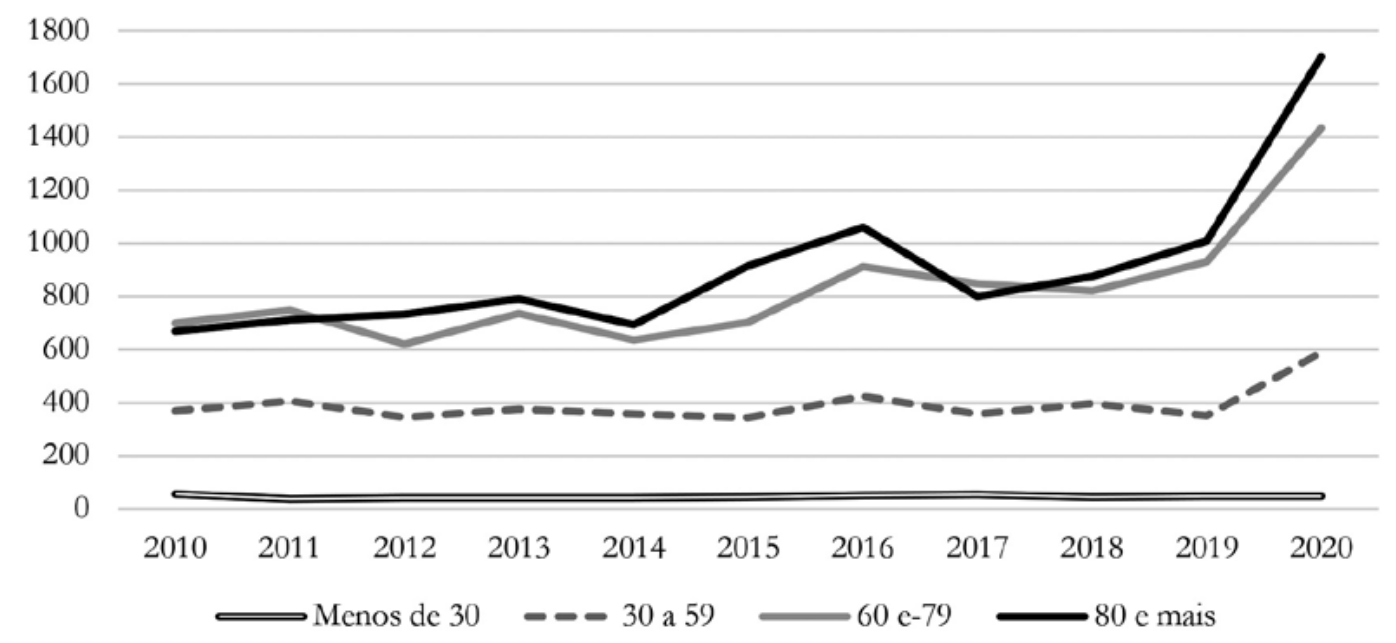

\begin{tabular}{lrrrrrrrrrrr}
\hline Proporção de óbitos que ocorrem em domicílios, segundo faixa etária e ano. \\
\hline Ano & 2010 & 2011 & 2012 & 2013 & 2014 & 2015 & 2016 & 2017 & 2018 & 2019 & 2020 \\
\hline Menos de 30 anos & 4,3 & 3,1 & 3,3 & 3,5 & 3,6 & 3,9 & 3,8 & 4,5 & 3,7 & 4,0 & 4,4 \\
30 a 59 & 9,8 & 10,8 & 9,6 & 10,1 & 10,5 & 9,9 & 11,2 & 10,4 & 11,7 & 9,7 & 10,7 \\
60 e-79 & 11,9 & 12,4 & 10,4 & 11,9 & 10,8 & 10,6 & 12,6 & 13,0 & 12,4 & 12,7 & 12,6 \\
80 e mais & 15,4 & 16,0 & 16,0 & 16,3 & 15,4 & 16,2 & 17,6 & 15,5 & 16,2 & 16,9 & 21,3 \\
\hline
\end{tabular}

Fonte: SMS/SUBPAV/SVS/CAS/GTDV - Sistema de Informações sobre Mortalidade (SIM). Dados sujeitos a revisão. Os dados utilizados referem-se aos meses de abril a junho.

Figura 1. Número de óbitos geral e em domicílios e proporção de óbitos em domicílios, segundo faixa etária e ano no município do Rio de janeiro, 2020. 
Tabela 1. Número e distribuição percentual dos óbitos de idosos no Rio de Janeiro (estado e município) nos meses de abril a junho do triênio (média de 2017 a 2019) e 2020, segundo local de ocorrência do óbito e diferença absoluta e percentual no período.

\begin{tabular}{|c|c|c|c|c|}
\hline & & \multicolumn{2}{|c|}{ Total de óbitos } & $\begin{array}{c}\text { Excesso de } \\
\text { Mortalidade em } 2020\end{array}$ \\
\hline \multirow{2}{*}{\multicolumn{2}{|c|}{ Local de ocorrência do óbito }} & $\begin{array}{c}\text { Média } \\
(2017-2019)\end{array}$ & 2020 & Diferença \\
\hline & & $\mathrm{N}(\%)$ & $\mathrm{N}(\%)$ & $\mathrm{N}(\%)$ \\
\hline \multicolumn{5}{|c|}{ Estado do Rio de Janeiro } \\
\hline \multicolumn{2}{|c|}{ Total óbitos de idosos } & $25.260(100)$ & $34.475(100)$ & $9.215(36,5)$ \\
\hline \multirow{6}{*}{$\begin{array}{l}\text { Local de } \\
\text { ocorrência }\end{array}$} & Hospital & $17.468(69,2)$ & $22.878(66,4)$ & $5.410(3,0)$ \\
\hline & Outro estabelecimento de saúde & $3.603(14,3)$ & $5.280(15,3)$ & $1.677(46,5)$ \\
\hline & Domicílio & $3.756(14,9)$ & $5.795(16,8)$ & $2.039(54,3)$ \\
\hline & Via pública & $69(0,3)$ & $71(0,2)$ & $2(3,4)$ \\
\hline & Outros & $359(1,4)$ & $446(1,3)$ & $87(24,3)$ \\
\hline & Ignorado ou não informado & $4(0,0)$ & $5(0,0)$ & $1(15,4)$ \\
\hline \multicolumn{5}{|c|}{ Município do Rio de Janeiro } \\
\hline \multicolumn{2}{|c|}{ Total óbitos de idosos } & $12.325(100)$ & $19.348(100)$ & $7.023(57)$ \\
\hline \multirow{6}{*}{$\begin{array}{l}\text { Local de } \\
\text { ocorrência }\end{array}$} & Hospital & $8.749(71)$ & $13.070(67,6)$ & $4.321(49,4)$ \\
\hline & Outro estabelecimento de saúde & $1.575(12,8)$ & $2.805(14,5)$ & $1.230(78,1)$ \\
\hline & Domicílio & $1.760(14,3)$ & $3.135(16,2)$ & $1.375(78,2)$ \\
\hline & Via pública & $13(0,1)$ & $28(0,1)$ & $15(115,4)$ \\
\hline & Outros & $227(1,8)$ & $307(1,6)$ & $80(35,4)$ \\
\hline & Ignorado ou não informado & $2(0,0)$ & $3(0,0)$ & $1(80)$ \\
\hline
\end{tabular}

Fonte: SMS/SUBPAV/SVS/CAS/GTDV - Sistema de Informações sobre Mortalidade (SIM). Dados sujeitos a revisão. Os dados utilizados referem-se aos meses de abril a junho.

Tabela 2. Número, distribuição proporcional e diferença percentual e absoluta dos óbitos de idosos que ocorreram no domicilio no Município do Rio de Janeiro, segundo grupos de causas da CID-10, nos meses de abril a junho do triênio 2017-2019 e 2020.

\begin{tabular}{lcccccc}
\hline & \multicolumn{2}{c}{ Total de óbitos } & \multicolumn{2}{c}{$\begin{array}{c}\text { Excesso de Mortalidade } \\
\text { em 2020 }\end{array}$} & \multicolumn{2}{c}{ Proporção } \\
\cline { 2 - 7 } Grupos de causas (CID-10) & $\begin{array}{c}\text { Média } \\
(2017-2019)\end{array}$ & 2020 & $\begin{array}{c}\text { Diferença } \\
\text { Absoluta }\end{array}$ & $\begin{array}{c}\text { Diferença } \\
\text { Percentual }\end{array}$ & $\begin{array}{c}\text { Média } \\
(2017-2019)\end{array}$ & 2020 \\
\hline Total de Óbitos & 1760 & 3135 & 1375 & 78,1 & 100,0 & 100,0 \\
\hline $\begin{array}{l}\text { I. Algumas doenças infecciosas e } \\
\text { parasitárias }\end{array}$ & 12 & 99 & 87 & 725,0 & 0,7 & 3,2 \\
$\begin{array}{l}\text { B34 Doenças por vírus, de localização } \\
\text { não especificada }\end{array}$ & 0 & 76 & 76 & $*$ & 0,0 & 2,4 \\
$\begin{array}{l}\text { II. Neoplasias (tumores) } \\
\text { C18 Neoplasia maligna do cólon }\end{array}$ & 152 & 330 & 178 & 117,1 & 8,6 & 10,5 \\
$\begin{array}{l}\text { C34 Neoplasia maligna dos brônquios e } \\
\text { dos pulmões }\end{array}$ & 10 & 21 & 11 & 110,0 & 0,6 & 0,7 \\
C50 Neoplasia maligna da mama & 23 & 43 & 20 & 87,0 & 1,3 & 1,4 \\
C61 Neoplasia maligna da próstata & 13 & 30 & 17 & 130,8 & 0,7 & 1,0 \\
& 16 & 46 & 30 & 187,5 & 0,9 & 1,5 \\
\hline
\end{tabular}


Continuação da Tabela 2

\begin{tabular}{|c|c|c|c|c|c|c|}
\hline \multirow{2}{*}{ Grupos de causas (CID-10) } & \multicolumn{2}{|c|}{ Total de óbitos } & \multicolumn{2}{|c|}{$\begin{array}{c}\text { Excesso de Mortalidade } \\
\text { em } 2020 \\
\end{array}$} & \multicolumn{2}{|c|}{ Proporção } \\
\hline & $\begin{array}{c}\text { Média } \\
(2017-2019)\end{array}$ & 2020 & $\begin{array}{l}\text { Diferença } \\
\text { Absoluta }\end{array}$ & $\begin{array}{l}\text { Diferença } \\
\text { Percentual }\end{array}$ & $\begin{array}{c}\text { Média } \\
(2017-2019)\end{array}$ & 2020 \\
\hline $\begin{array}{l}\text { III. Doenças do sangue e dos órgãos } \\
\text { hematopoiéticos e alguns transtornos } \\
\text { imunitários }\end{array}$ & 4 & 10 & 6 & 150,0 & 0,2 & 0,3 \\
\hline D50; D53; D62; D64 Anemias & 3 & 9 & 6 & 200,0 & 0,2 & 0,3 \\
\hline $\begin{array}{l}\text { IV. Doenças endócrinas, nutricionais e } \\
\text { metabólicas }\end{array}$ & 133 & 252 & 119 & 89,5 & 7,6 & 8,0 \\
\hline E14 Diabetes mellitus não especificado & 96 & 184 & 88 & 91,7 & 5,5 & 5,9 \\
\hline V. Transtornos mentais e comportamentais & 18 & 46 & 28 & 155,6 & 1,0 & 1,5 \\
\hline F03 Demência não especificada & 7 & 13 & 6 & 85,7 & 0,4 & 0,4 \\
\hline $\begin{array}{l}\text { F10 Transtornos mentais e } \\
\text { comportamentais devidos ao uso de } \\
\text { álcool }\end{array}$ & 3 & 14 & 11 & 366,7 & 0,2 & 0,4 \\
\hline VI. Doenças do sistema nervoso & 87 & 139 & 52 & 59,8 & 5,0 & 4,4 \\
\hline G20 Doença de Parkinson & 16 & 26 & 10 & 62,5 & 0,9 & 0,8 \\
\hline G30 Doença de Alzheimer & 59 & 99 & 40 & 67,8 & 3,4 & 3,2 \\
\hline IX. Doenças do aparelho circulatório & 956 & 1274 & 318 & 33,3 & 54,3 & 40,6 \\
\hline I10; I11 Hipertensão & 153 & 364 & 211 & 137,9 & 8,7 & 11,6 \\
\hline I21 Infarto agudo do miocárdio & 469 & 560 & 91 & 19,4 & 26,6 & 17,9 \\
\hline I50 Insuficiência cardíaca & 32 & 90 & 58 & 181,3 & 1,8 & 2,9 \\
\hline $\begin{array}{l}\text { I64 Acidente vascular cerebral, não } \\
\text { especificado como hemorrágico ou } \\
\text { isquêmico }\end{array}$ & 34 & 63 & 29 & 85,3 & 1,9 & 2,0 \\
\hline X. Doenças do aparelho respiratório & 146 & 164 & 18 & 12,3 & 8,3 & 5,2 \\
\hline J43 Enfisema & 11 & 17 & 6 & 54,5 & 0,6 & 0,5 \\
\hline $\begin{array}{l}\text { J44 Outras doenças pulmonares } \\
\text { obstrutivas crônicas }\end{array}$ & 17 & 24 & 7 & 41,2 & 1,0 & 0,8 \\
\hline $\begin{array}{l}\text { J96 Insuficiência respiratória não } \\
\text { classificada em outra parte }\end{array}$ & 5 & 25 & 20 & 400,0 & 0,3 & 0,8 \\
\hline XI. Doenças do aparelho digestivo & 33 & 38 & 5 & 15,2 & 1,9 & 1,2 \\
\hline $\begin{array}{l}\text { XII. Doenças da pele e do tecido } \\
\text { subcutâneo }\end{array}$ & 5 & 12 & 7 & 140,0 & 0,3 & 0,4 \\
\hline $\begin{array}{l}\text { XIII.Doenças do sistema } \\
\text { osteomuscular e do tecido conjuntivo }\end{array}$ & 5 & 15 & 10 & 200,0 & 0,3 & 0,5 \\
\hline XIV. Doenças do aparelho geniturinário & 18 & 39 & 21 & 116,7 & 1,0 & 1,2 \\
\hline $\begin{array}{l}\text { XVIII.Sintomas, sinais e achados anormais } \\
\text { de exames clínicos e de laboratório, não } \\
\text { classificados em outra parte }\end{array}$ & 152 & 662 & 510 & 335,5 & 8,7 & 21,1 \\
\hline $\begin{array}{l}\text { XX. Causas externas de morbidade e } \\
\text { mortalidade }\end{array}$ & 37 & 55 & 18 & 48,6 & 2,1 & 1,8 \\
\hline $\begin{array}{l}\text { Y34 Fatos ou eventos não especificados } \\
\text { e intenção não determinada }\end{array}$ & 9 & 26 & 17 & 188,9 & 0,5 & 0,8 \\
\hline
\end{tabular}

*Proporção atribuível à Covid-19

Fonte: SMS/SUBPAV/SVS/CAS/GTDV - Sistema de Informações sobre Mortalidade (SIM). Dados sujeitos a revisão. Os dados utilizados referem-se aos meses de abril a junho. 
Tabela 3. Número, proporção e diferença percentual de óbitos gerais e ocorridos em domićlios entre abril e junho no triênio 2017-2019 e 2020, segundo características sociodemográficas, no município do Rio de Janeiro.

\begin{tabular}{|c|c|c|c|c|c|c|}
\hline \multirow{3}{*}{ Variáveis e categorias } & \multicolumn{3}{|c|}{ Óbitos ocorridos em domicílio } & \multicolumn{3}{|c|}{ Todos os óbitos } \\
\hline & $\begin{array}{c}\text { Média } \\
(2017-2019)\end{array}$ & 2020 & Diferença & $\begin{array}{c}\text { Média } \\
(2017-2019)\end{array}$ & 2020 & Diferença \\
\hline & $\mathrm{N}(\%)$ & $\mathrm{N}(\%)$ & $\%$ & $\mathrm{~N}(\%)$ & $\mathrm{N}(\%)$ & $\%$ \\
\hline Total & $1.760(100)$ & $3.138(100)$ & 78,3 & $12.324(100)$ & $19.501(100)$ & 58,2 \\
\hline \multicolumn{7}{|l|}{ Sexo } \\
\hline Masculino & $807(45,9)$ & $1.529(48,7)$ & 89,4 & $5.607(45,5)$ & $9.748(50)$ & 73,9 \\
\hline Feminino & $952(54,1)$ & $1.609(51,3)$ & 69,0 & $6.718(54,5)$ & $9.753(50)$ & 45,2 \\
\hline \multicolumn{7}{|l|}{ Raça/cor } \\
\hline Branca & $1.172(66,6)$ & $2.038(64,9)$ & 73,9 & $7.569(61,4)$ & $11315(58)$ & 49,5 \\
\hline Preta & $170(9,7)$ & $356(11,3)$ & 109,0 & $1.391(11,3)$ & $2587(13,3)$ & 86,0 \\
\hline Amarela & $3(0,2)$ & $5(0,2)$ & 66,7 & $19(0,2)$ & $40(0,2)$ & 114,3 \\
\hline Parda & $406(23,1)$ & $722(23)$ & 77,7 & $3.251(26,4)$ & $5393(27,7)$ & 65,9 \\
\hline Indígena & $1(0,1)$ & $0(0)$ & $-100,0$ & $4(0)$ & $3(0)$ & $-18,2$ \\
\hline Não informado & $8(04)$ & $17(0,5)$ & 121,7 & $91(0,7)$ & $163(08)$ & 79,1 \\
\hline \multicolumn{7}{|l|}{ Estado Civil } \\
\hline Solteiro & $395(22,4)$ & $629(20)$ & 59,2 & $2.409(19,5)$ & $3640(18,7)$ & 51,1 \\
\hline Casado & $4961(28,2)$ & $906(28,9)$ & 82,5 & $4.061(33)$ & $7050(36,2)$ & 73,6 \\
\hline Viúvo & $677(38,5)$ & $1.210(38,6)$ & 78,8 & $4.529(36,8)$ & $6546(33,6)$ & 44,5 \\
\hline Separado judicialmente & $162(9,2)$ & $281(9)$ & 73,5 & $1.082(8,8)$ & $1734(8,9)$ & 60,2 \\
\hline União consensual & $10(0,6)$ & $43(1,4)$ & 330,0 & $85(0,7)$ & $205(1,1)$ & 140,2 \\
\hline Não informado & $3(0,2)$ & $6(0,2)$ & 80,0 & $45(0,4)$ & $83(0,4)$ & 85,8 \\
\hline Ignorado & $16(0,9)$ & $63(2)$ & 285,7 & $113(0,9)$ & $243(1,2)$ & 115,7 \\
\hline \multicolumn{7}{|l|}{ Escolaridade } \\
\hline Nenhuma & $106(6)$ & $216(6,9)$ & 103,1 & $811(6,6)$ & $1163(6)$ & 43,4 \\
\hline 1-3 anos & $387(22)$ & $757(24,1)$ & 95,6 & $3.567(28,9)$ & $4960(25,4)$ & 39,0 \\
\hline 4-7 anos & $375(21,3)$ & $503(16)$ & 34,0 & $2.582(21)$ & $3825(19,6)$ & 48,1 \\
\hline 8-11 anos & $386(22)$ & $705(22,5)$ & 82,5 & $2.707(22)$ & $4871(25)$ & 80,0 \\
\hline 12 anos e mais & $235(13,4)$ & $453(14,4)$ & 92,5 & $1.478(12)$ & $2689(13,8)$ & 82,0 \\
\hline Não informado & $29(1,6)$ & $43(1,4)$ & 48,3 & $109(0,9)$ & $315(1,6)$ & 189,0 \\
\hline Ignorado & $240(13,7)$ & $461(14,7)$ & 91,8 & $1.070(8,7)$ & $1678(8,6)$ & 56,8 \\
\hline
\end{tabular}

Fonte: SMS/SUBPAV/SVS/CAS/GTDV - Sistema de Informações sobre Mortalidade (SIM). Dados sujeitos a revisão. Os dados utilizados referem-se aos meses de abril a junho.

Em relação ao estado civil, verificou-se um aumento mais expressivo do número de óbitos de viúvo que ocorreram no domicílio $(78,8 \%)$, em relação aos óbitos em todos os locais de ocorrência $(44,5 \%)$. O mesmo pode ser observado, ainda que em menor escala, entre os separados, com aumento de $73,5 \%$ da morte domiciliar e $60,2 \%$ em todos os locais de ocorrência. Novamente destaca-se o crescimento acentuado de óbitos com informação sociodemográfica ignorada, especialmente no domicílio $(285,7 \%)$.

Quanto à escolaridade, verificou-se um aumento percentual acentuado na mortalidade domiciliar entre pessoas com escolaridade mais baixa, especialmente nas categorias "Nenhuma escolaridade" (aumento percentual de 103,1\%) e "1 a 3 anos de estudo" $(95,6 \%)$, em relação aos óbitos em todos os locais 
de ocorrência, que tiveram respectivamente $43,4 \%$ e 39,0\% de aumento. A informação de escolaridade "ignorada" ou "não informada" é elevada tanto na mortalidade domiciliar quanto nos demais locais.

\section{DISCUSSÃO}

A proporção de óbitos domiciliares de pessoas idosas aumentou expressivamente nos meses de abril a junho de 2020 quando comparado com a média desses meses no triênio anterior, com destaque para o grupo de 80 anos e mais, tanto no Estado quanto no Município do Rio de Janeiro. Apesar de as principais causas de mortalidade domiciliar não serem alteradas, podemos notar aumentos proporcionais relevantes tanto para hipertensão, quanto para diabetes. Também foi observado crescimento acentuado das mortes por doenças infecciosas e parasitárias. Entre as doenças do aparelho circulatório, destaca-se o crescimento da insuficiência respiratória não classificada. Os óbitos por causa mal definida tiveram um aumento proporcional de $335,5 \%$. Quanto às características sociodemográficas da mortalidade domiciliar no município, verificou-se maior aumento percentual entre os homens, população com raça/cor declarada negra, pessoas viúvas e de baixa escolaridade.

Uma hipótese que pode explicar o excesso de mortalidade domiciliar é a própria pandemia, que aumentou a procura por leitos públicos. Outros estudos sobre a mortalidade domiciliar na população geral trabalham com hipótese explicativa similar ${ }^{3,4,9}$. Devido à incipiente oferta de atendimento da rede assistencial pública de saúde no estado e no município do Rio de Janeiro, é provável que muitos pacientes tenham retornado às suas residências sem o cuidado necessário. A atenção primária à saúde (APS) passa pelo mesmo problema.

A busca por leitos e atendimento público pode ter sido prejudicada pela piora dos indicadores de cobertura no Rio de Janeiro. De acordo com Martins e colaboradores (2019), os problemas de acesso e qualidade da rede hospitalar pública se intensificaram no estado, alertando para um estágio de crise no cuidado hospitalar ${ }^{13}$.Estudos mostram que a situação do município é similar, onde verificou-se redução da população coberta pela APS $^{14,15}$, da realização de procedimentos, bem como recursos humanos e materiais essenciais, tais como leitos e equipes de saúde da família ${ }^{15}$.

Além deste fator, o aumento dos óbitos domiciliares também pode estar ligado à redução assistencial regular decorrente do distanciamento social. A pandemia implicou alterações no funcionamento do sistema de saúde, dentre as quais destaca-se a interrupção de atividades não essenciais ou eletivas. Isso, aliado ao medo de contaminação pelos pacientes, teria provocado mudanças importantes na dinâmica de atendimento de serviços hospitalares ${ }^{16}$. Essas alterações teriam acarretado uma diminuição do fluxo de pacientes nos hospitais, inclusive daqueles com indicação de atendimento emergencial, o que poderia estar colaborando com o aumento de mortes em domicílio ${ }^{9,17}$. O achado da concentração do excesso de mortalidade entre as faixas etárias mais altas é corroborado por estudos nacionais e internacionais ${ }^{18-20}$, e explicado pela maior vulnerabilidade desta faixa etária.

O aumento dos óbitos por hipertensão e diabetes também são causa de preocupação visto que ambas são consideradas causas evitáveis de mortalidade ${ }^{21}$ e internação ${ }^{22}$. Sabe-se que a alta cobertura da APS está associada com a diminuição de óbitos evitáveis ${ }^{23 .}$ Ademais, com uma APS adequada, a população tem maior acesso à prevenção e ao tratamento de diversos agravos, o que possivelmente evitaria grande parte dos óbitos que ocorrem em domicílio. Mesmo com os desafios que a pandemia trouxe para o sistema de saúde ${ }^{17}$, espera-se que num cenário com uma ação articulada entre as equipes de saúde da família, com suficientes ACS, e assistência social, fosse possível ter evitado um copioso número de óbitos de idosos, independentemente do seu local de ocorrência.

Em 2020, a diferença percentual dos óbitos por doenças infecciosas e parasitárias em relação à média do triênio anterior sobressai. O número que antes era de 12 óbitos, chega quase cem, um aumento de $702 \%$.

Outra questão importante levantada nos resultados é o aumento de mortalidade por causa básica mal definida. A falta de conhecimento da causa básica do óbito pode estar relacionada com a carência de testes da Covid-19 e a falta de rede de atendimento adequada a casos graves. Assim como em grande parte 
do país, o município do Rio de Janeiro padeceu com a quantidade incipiente de testes para detecção do novo coronavírus e mesmo quando eram realizados, muitos pacientes faleciam antes de terem seus resultados liberados ${ }^{24}$. Com isso, é possível e provável que grande parte dos óbitos registrados como mal definidos possam ser atribuídos à Covid-19.

Além disso, é importante notar que a Covid-19 é uma doença sistêmica, que pode afetar diversos órgãos do corpo humano, e cujas interações ainda não foram completamente compreendidas e estudadas. Por este motivo, o aumento de óbitos provocados pela Covid-19 pode estar diluído em outras causas ${ }^{25}$.

Os resultados que apontam maior proporção de morte para homens vão no mesmo sentido de estudos recentes em diversos países ${ }^{26-28}$, que mostram letalidade mais alta entre homens, mesmo quando ajustado por idade e comorbidades.

Pessoas idosas viúvas apresentaram maior proporção de mortalidade na residência do que as com outra situação conjugal. Isso pode se dever a menor rede de apoio social dessas pessoas. Por isso estudos suplementares são necessários para compreender os motivos do maior risco para esse grupo populacional.

O maior percentual de aumento dos óbitos domiciliares na população idosa negra, em relação às demais cores da pele, pode ser explicado pelas fortes desigualdades raciais no país. O racismo estrutural vem historicamente se refletindo em piores indicadores de acesso aos serviços e situação de saúde, o que consequentemente torna o impacto da pandemia especialmente oneroso para esse grupo ${ }^{29}$

Foi encontrada maior proporção de morte domiciliar para pessoas com menor escolaridade, esse achado é similar ao de outros estudos, tanto no Brasil quanto no exterior ${ }^{20,26,27,30}$. Diversos artigos já apontaram que o menor nível de escolaridade é uma barreira de acesso aos serviços de saúde, tanto porque esta população em geral habita regiões com baixa infraestrutura de serviços básicos ${ }^{20,31}$, como porque pode reduzir a compreensão do sistema, dificultar o reconhecimento de situações de risco e consequentemente reduzindo a utilização dos serviços de saúde ${ }^{20}$.
Para a população idosa os impactos das diferenças socioeconômicas são potencializados. As desigualdades sociais, na saúde e nas condições de vida, fazem com que grupos vulneráveis principalmente indígenas, negros e com baixa renda - experimentem um processo de envelhecimento com sobreposição de riscos, que os tornam mais vulneráveis $^{20,32}$. A pandemia de Covid-19 no Brasil tem deixado claro o impacto dessas diferenças e os desafios de gestão do sistema de saúde neste contexto.

Como limitação importante deste artigo devemos mencionar a impossibilidade de determinar se a morte domiciliar ocorreu em residência particular ou coletiva. Os sistemas de informação em saúde brasileiros não contêm dados desagregados sobre esse tipo de residência, uma vez que estão vinculadas à assistência social e não à saúde. Não foram encontrados dados oficiais sobre mortalidade em ILPI, seja por Covid-19 ou por outras causas. Outra limitação refere-se a possível subnotificações dos óbitos, no entanto, isso não compromete a qualidade do estudo, uma vez que o SIM tem alta cobertura no município do Rio de Janeiro.

As ILPI são reconhecidas internacionalmente como como espaço de alto risco para a transmissão e mortalidade pela Covid-19. Estudos conduzidos em países com adequada vigilância epidemiológica mostraram que metade dos óbitos atribuídos à Covid-19 ocorrem nessas instituições?. Isso ocorre porque, em geral, elas concentram indivíduos com idade avançada, mais vulneráveis e que já possuem outras morbidades. No Brasil, a despeito das orientações e estratégias de prevenção contra Covid-19 em ILPI ${ }^{33-35}$, ainda não foi possível avaliar seus desdobramentos.

\section{CONCLUSÃO}

O aumento da mortalidade domiciliar encontrado no município do Rio de Janeiro pode estar associado a efeitos da pandemia de Covid-19, conforme apontado na literatura. Além disso, o aumento de mortes com causa básica mal definida pode estar associada à pandemia de Covid-19 em razão da falta de testes; dificuldade de acesso a serviços de saúde. 
A análise mostrou que a mortalidade residencial por Covid-19 tem relação com sexo masculino, viúvos e faixa etária mais elevada. Apesar de a maior vulnerabilidade entre os mais velhos ser conhecida, estudos adicionais são importantes para entender os diferenciais de sexo e arranjos matrimoniais.

Os dados também mostraram ligação entre raça/ cor negra e menor nível de escolaridade e maior chance de mortalidade na residência durante a pandemia de Covid-19. Isso acontece em razão de uma sobreposição de riscos durante o ciclo vital, os quais fazem com que as pessoas nesses grupos sociais envelheçam mais vulneráveis. Contudo, estudos suplementares sobre trabalho e emprego

\section{REFERÊNCIAS}

1. Fundação Oswaldo Cruz, Instituto de Comunicação e Informação Científica e Tecnológica em Saúde (ICICT). MonitoraCovid-19 [Internet]. Rio de Janeiro: FIOCRUZ; 2020 [acesso em 22 fev. 2021]. Disponível em: https://bigdata-covid19.icict.fiocruz.br/.

2. Sistema de Indicadores de Saúde e Acompanhamento de Políticas do Idoso (SISAP-Idoso) [Internet]. Rio de Janeiro: FIOCRUZ; 2020 [acesso em 29 set. 2020]. Disponível em: https://sisapidoso.icict.fiocruz.br/.

3. Observatório Covid-19 [Internet]. Rio de Janeiro: FIOCRUZ; 2020. Nota Técnica 11: Óbitos desassistidos no Rio de Janeiro. Análise do excesso de mortalidade e impacto da Covid-19. 2020 [acesso em 28 ago. 2020]. Disponível em: https://bigdatacovid19.icict.fiocruz.br/nota_tecnica_11.pdf.

4. Universidade Estadual do Rio de Janeiro, Instituto de Medicina Social. Nota Técnica: Uma análise dos óbitos ocorridos nos domicílios do estado do Rio de Janeiro [Internet]. Rio de Janeiro: UERJ; 2020 [acesso em 28 ago. 2020]. Disponível em: https://www.ims. uerj.br/wp-content/uploads/2020/07/NT-obitos-nosdomicilios-mar-mai-RJ-vfinal.pdf.

5. Menezes RA, Barbosa PC. A construção da "boa morte" em diferentes etapas da vida: reflexões em torno do ideário paliativista para adultos e crianças. Ciênc Saúde Colet. 2013;18(9):2653-62. Disponível em: https://www.scielo.br/pdf/csc/v18n9/v18n9a20.pdf.

6. Marcucci FCI, Cabrera MAS. Death in hospital and at home: population and health policy influences in Londrina, State of Paraná, Brazil (1996-2010). Ciênc Saúde Colet. 2015;20(3):833-40. Disponível em: https://doi.org/10.1590/1413-81232015203.04302014. e condições de moradia desses indivíduos seriam importantes para que pudéssemos compreender melhor os mecanismos por trás da mortalidade domiciliar durante a pandemia de Covid-19.

Apesar da relevância do debate sobre as ILPÌ no contexto da pandemia, a invisibilidade do tema nos sistemas de informação impediu que sua análise fosse aprofundada neste estudo. Apesar de não ter sido possível identificar o tipo do domicílio em que ocorreu o óbito (particular ou coletivo), apresenta-se a distribuição dos óbitos por variáveis demográficas, socioeconômicas e por causas de óbito.

Editado por: Maria Helena Rodrigues Galvão
7. Boas PJFV, Bremenkamp MG, Roriz Filho J S, Kairalla MC, Gomes DCA, de Mello RGB, et al. Recommendations for the prevention and control of coronavirus infections (SARS-CoV-2) in long term care facilities. Geriatr Gerontol Aging. 2020;14(2):134-7. Disponível em: https://doi. org/10.5327/Z2447-2123202020142ESP3.

8. D’Adamo H, Yoshikawa T, Ouslander JG.

Coronavirus Disease 2019 in Geriatrics and LongTerm Care: The ABCDs of COVID-19. J Am Geriatr Soc. 2020;68(5):912-7. Disponível em: https://doi. org/10.1111/jgs.16445.

9. Rafael RMR, Bello R, Jalles AP, Mangabeira RA, Maçana SC, Paula MCR. COVID-19: monitoramento de síndromes respiratórias e óbitos domiciliares no Serviço de Atendimento Móvel de Urgência. Res Soc Dev. 2020;9(9):e914998044. Disponível em: https:// doi.org/10.33448/rsd-v9i9.8044 .

10. Rafael RMR, Neto M, Depret DG, Gil AC, Fonseca MHS, Souza-Santos R, et al. Effect of income on the cumulative incidence of COVID-19: an ecological study. Rev Latinoam Enferm. 2020;28:e3344. Disponível em: https://doi.org/10.1590/1518-8345.4475.3344.

11. Rio de Janeiro (Município). Secretaria Municipal de Saúde. Saúde-Rio: TABNET Municipal [Internet]. 2020 [acesso em 15 out. 2020]. Disponível em: http:// tabnet.rio.rj.gov.br/tabnet/.

12. Kanso S, Romero DE, Leite IC, de Moraes EN. Diferenciais geográficos, socioeconômicos e demográficos da qualidade da informação da causa básica de morte dos idosos no Brasil. Cad Saúde Pública. 2011;27(7):1323-39. Disponível em: https:// www.scielo.br/pdf/csp/v27n7/08.pdf. 
13. Martins M, Lima SML, de Andrade CLT, Portela MC. Indicadores hospitalares de acesso e efetividade e crise econômica: análise baseada nos dados do Sistema Único de Saúde, Brasil e estados da região Sudeste, 2009-2018. Ciênc Saúde Colet. 2019;24:454154. Disponível em: https://doi.org/10.1590/1413812320182412.25262019 .

14. O’Dwyer G, Graever L, Britto FA, Menezes T, Konder MT. A crise financeira e a saúde: o caso do município do Rio de Janeiro, Brasil. Ciênc Saúde Colet. 2019;24:4555-68. Disponível em: https://doi. org/10.1590/1413-812320182412.23212019 .

15. Melo EA, de Mendonça MM, Teixeira M. A crise econômica e a atenção primária à saúde no SUS da cidade do Rio de Janeiro, Brasil. Ciênc Saúde Colet. 2019;24:4593-8. Disponível em: https://doi. org/10.1590/1413-812320182412.25432019.

16. Oliveira M, Sousa P, Sousa L, Mendes C, Labucha M, Macedo F. A Cardiologia na Pandemia COVID-19: que Impacto na atividade clínica? Gaz Méd [Internet]. 2020;7(2):1-10. Disponível em: https://doi. org/10.29315/gm.v7i2.348.

17. Alves THE, de Souza TA, Silva SA, Ramos NA, de Oliveira SV. Análise de óbitos domiciliares e hospitalares por causas respiratórias e cardiovasculares durante à pandemia da COVID-19 em Minas Gerais. Scielo Preprints. 2020 [Postado em 02 ago. 2020; acesso em 18 ago. 2020]: 10 p. Disponível em: https:// doi.org/10.1590/SciELOPreprints.1021.

18. Shahid Z, Kalayanamitra R, McClafferty B, Kepko D, Ramgobin D, Patel R, et al. COVID-19 and Older Adults: What We Know. J Am Geriatr Soc. 2020;68(5):926-9. Disponível em: https://doi. org/10.1111/jgs.16472.

19. Lithander FE, Neumann S, Tenison E, Lloyd K, Welsh TJ, Rodrigues JCL, et al. COVID-19 in older people: a rapid clinical review. Age Ageing. 2020;49(4):501-15. Disponível em: https://doi. org/10.1093/ageing/afaa093.

20. Barbosa IR, Galvão MHR, de Souza TA, Gomes $\mathrm{SM}$, Medeiros AA, de Lima KC, et al. Incidence of and mortality from COVID-19 in the older Brazilian population and its relationship with contextual indicators: an ecological study. Rev Bras Geriatr Gerontol. 2020];23(1):1-9. Disponível em: https://doi. org/10.1590/1981-22562020023.200171.

21. Malta DC, Duarte EC, de Almeida MF, Dias MAS, de Morais Neto OL, de Moura L, et al. Lista de causas de mortes evitáveis por intervenções do Sistema Único de Saúde do Brasil. Epidemiol Serv Saúde. 2007;16(4):233-44. Disponível em: http://scielo.iec. gov.br/pdf/ess/v16n4/v16n4a02.pdf.
22. Marques AP, Montilla DER, de Almeida WS, de Andrade CLT. Hospitalization of older adults due to ambulatory care sensitive conditions. Rev Saúde Pública. 2014;48(5):817-26. Disponível em: https:// doi.org/10.1590/S0034-8910.2014048005133.

23. Malta DC, Duarte EC. Causas de mortes evitáveis por ações efetivas dos serviços de saúde: uma revisão da literatura. Ciênc Saúde Colet. 2007;12:765-76. Disponível em: https://www.scielo.br/pdf/csc/ v12n3/27.pdf.

24. França EB, Ishitani LH, Teixeira RA, de Abreu DMX, Corrêa PRL, Marinho F, et al. Óbitos por COVID-19 no Brasil: quantos e quais estamos identificando? Rev Bras Epidemiol. 2020;23:e200053. Disponível em: https://doi.org/10.1590/1980549720200053.

25. Campos MR, Schramm JMA, Emmerick ICM, Muzy J, de Avelar FG, Pimentel TG. Carga de doença da COVID-19 e de suas complicações agudas e crônicas: reflexões sobre a mensuração (DALY) e perspectivas no Sistema Único de Saúde. Cad Saúde Pública. 2020;36(11):e00148920. Disponível em: https://doi. org/10.1590/0102-311X00148920.

26. Zhou F, Yu T, Du R, Fan G, Liu Y, Liu Z, et al. Clinical course and risk factors for mortality of adult inpatients with COVID-19 in Wuhan, China: a retrospective cohort study. Lancet. 2020;395(10229):1054-62. Disponível em: https://doi. org/10.1016/S0140-6736(20)30566-3.

27. Goyal P, Choi JJ, Pinheiro LC, Schenck EJ, Chen R, Jabri A, et al. Clinical Characteristics of Covid-19 in New York City. N Engl J Med. 2020:1-3. Disponível em: https://www.nejm.org/doi/pdf/10.1056/ NEJMc2010419?article'Tools=true.

28. Kragholm K, Andersen MP, Gerds TA, Butt JH, Østergaard L, Polcwiartek C, et al. Association Between Male Sex and Outcomes of Coronavirus Disease 2019 (COVID-19): a Danish Nationwide, Register-based Study. Clin Infect Dis. 2020:1-6. Disponível em: https://doi.org/10.1093/cid/ciaa924.

29. Goes EF, Ramos DO, Ferreira AJF, Goes EF, Ramos DO, Ferreira AJF. Desigualdades raciais em saúde e a pandemia da Covid-19. Trab Educ Saúde. 2020;18(3)19. Disponível em: https://www.scielo.br/pdf/tes/ v18n3/0102-6909-tes-18-3-e00278110.pdf.

30. Drefahl S, Wallace M, Mussino E, Aradhya S, Kolk $\mathrm{M}$, Brandén $\mathrm{M}$, et al. A population-based cohort study of socio-demographic risk factors for COVID-19 deaths in Sweden. Nat Commun. 2020;11(1):e5097. Disponível em: https://www.nature.com/articles/ s41467-020-18926-3.pdf. 
31. Stopa SR, Malta DC, Monteiro CN, Szwarcwald CL, Goldbaum M, Cesar CLG. Use of and access to health services in Brazil, 2013 National Health Survey. Rev Saúde Pública. 2017;51(Supp1):1-9. Disponível em: http://dx.doi.org/10.1590/s1518-8787.2017051000074.

32. De Oliveira BLCA, Thomaz EBAF, da Silva RA, de Oliveira BLCA, Thomaz EBAF, da Silva RA. The association between skin color/race and health indicators in elderly Brazilians: a study based on the Brazilian National Household Sample Survey (2008). Cad Saúde Pública. 2014;30(7):1438-52. Disponível em: https://doi.org/10.1590/0102-311X00071413.

33. Brasil. Ministério da Saúde. Nota técnica No 9/2020-COSAPI/CGCIVI/DAPES/SAPS/MS [Internet]. [acesso em 26 de ago. 2020]. Disponível em: https://idoso.mppr.mp.br/arquivos/File/ ILPI_NT_N_9_2020_COSAPI_CGCIVI_DAPES_ SAPS_MS.pdf
34. Agência Nacional de Vigilância Sanitária. Nota Técnica n. 05 de 2020 GVIMS/GGTES-ANVISA [Internet]. Orientações para prevenção e o controle de infecções [...]. 2020 [acesso em 26 ago. 2020]. Disponível em: https://www.gov.br/anvisa/pt-br/ centraisdeconteudo/publicacoes/servicosdesaude/ notas-tecnicas/nota-tecnica-no-05-2020-gvims-ggtesanvisa-orientacoes-para-a-prevencao-e-o-controle-deinfeccoes-pelo-novo-coronavirus-em-instituicoes-delonga-permanencia-para-idosos-ilpi.pdf/view.

35. Brasil. Portaria no 65, de 6 de maio de 2020. Aprova orientações e recomendações gerais aos gestores e trabalhadores do Sistema Único de Assistência Social dos estados, municípios e Distrito Federal quanto ao atendimento nos serviços de acolhimento de pessoas idosas ou com deficiência no contexto de emergência em saúde pública decorrente do novo Coronavírus, COVID-19. Diário Oficial da União. 07 maio 2020. Disponível em: https://www.in.gov.br/web/dou/-/ portaria-n-65-de-6-de-maio-de-2020-255614645. 